\title{
DYNAMICAL IMPLICATIONS OF DEEP HST IMAGING OF GLOBULAR CLUSTERS ${ }^{1}$
}

\author{
IVAN R. KING \\ Astronomy Department, University of California \\ Berkeley, CA 94720-3411, U.S.A.
}

\begin{abstract}
HST observations contribute in many ways to a better understanding of the dynamical nature of globular clusters. Unprecedentedly faint photometry gives new determinations of the numbers of low-mass stars. Cluster-to-cluster differences at the faint ends of the mass functions suggest differences in dynamical evolution. Mass segregation is clearly observed, from the envelope inward to the dense cluster center. The distribution of stars in the hitherto unresolved cores gives new data with which to test theories of core collapse, and these core profiles are also sensitive to the number of unseen remnant stars and binaries at the cluster center.
\end{abstract}

(Note: The results discussed in this paper come from a collaboration between the author, Adrienne Cool, Giampaolo Piotto, Craig Sosin, and Jay Anderson. Parts of this work are described elsewhere in this volume by Piotto et al., by Cool et al., and by Sosin \& King.)

\section{Introduction}

The Hubble Space Telescope offers two great new advantages in the study of globular clusters: it allows observing faint stars in the center, and it goes much fainter than we have gone from the ground.

The best ground-based resolving power is characterized by a full width at half maximum of a little better than 0.4 arcsec. By contrast, the central peak of the diffraction-limited HST image has a FWHM of 0.045 arcsec, almost a factor of ten times better. To be sure, some of the best HST

\footnotetext{
${ }^{1}$ Based on observations with the NASA/ESA Hubble Space Telescope, obtained at the Space Telescope Science Institute, which is operated by AURA, Inc., under NASA contract NAS5-26555.
} 


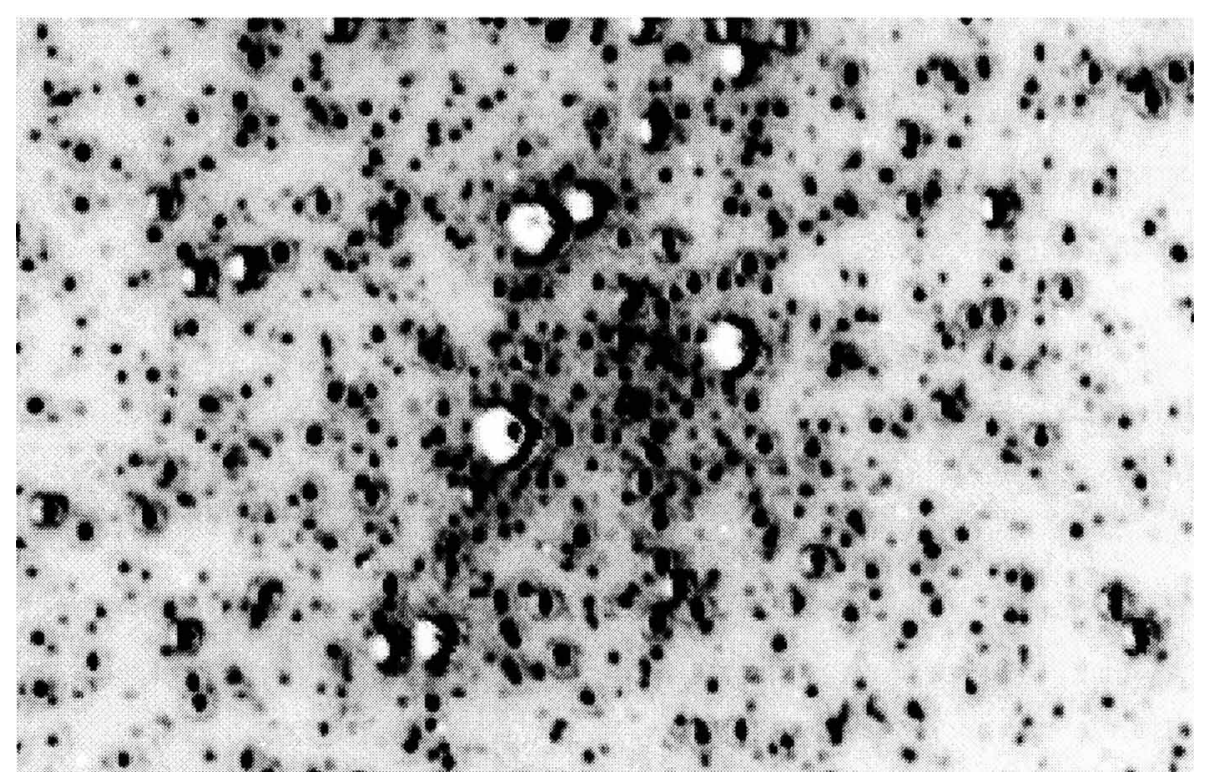

Figure 1. A 5"!6 63 "!5 part of an FOC image of the center of M15. The pixel size is 0.014 arcsec. The white areas are FOC saturation in the bright stars.

imaging is done with the Wide Field Camera, whose pixel is 0.1 arcsec, but this is still considerably better than we can do from the ground. Groundbased observers are now experimenting with aperture-synthesis techniques that will allow them to reach the diffraction limit, but this is still in the future, and will cover a much smaller area than the WFC field. By far the best tool we have today for imaging globular clusters is HST.

As an example, Figure 1 shows the center of M15, as imaged with HST's Faint Object Camera. Here we absolutely have to have the resolution of the FOC; the Planetary Camera, with 3 times as large a pixel, just doesn't have a high enough resolution.

As for faintness, my favorite comparison is between our HST result and a deep study done with the CTIO 4-meter (Fig. 2). The color scales are different, but the vertical scale of $V$ magnitudes is the same in both diagrams. We can do a lot with those extra 3 or 4 magnitudes of depth.

Since this is a dynamics meeting, I will center my emphasis on dynamics; but the data with which I will be dealing are photometric, so I will begin with a few words about photometric techniques, followed by a brief discussion of some of our results. 

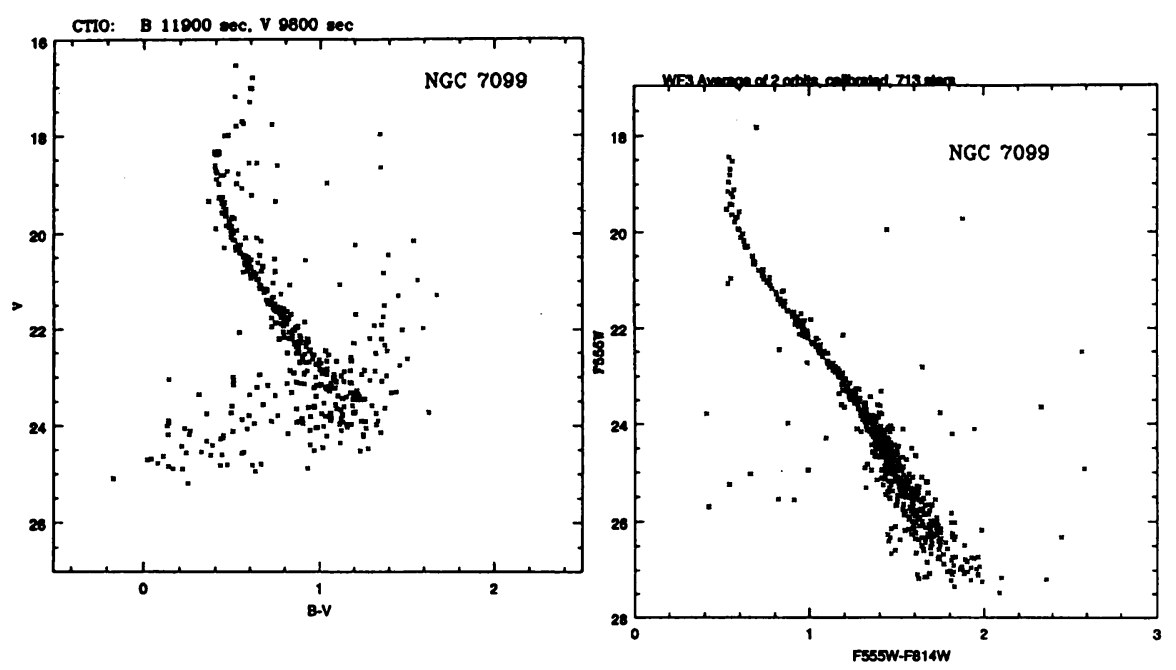

Figure 2. Comparison of ground-based (left) and HST CMDs of the same cluster. The color systems are different, but the two graphs have the same vertical $V$ scale, which shows how much fainter HST goes.

\section{Photometric Results}

Photometry with HST has turned out to be something of an art. We began by using DAOPHOT in what has become the standard way, but the results weren't very good. We then did a lot of experimenting and arrived at a method that was much more accurate. (For further details see Cool \& King 1995 , where it is explained that we later found that an unconventional use of DAOPHOT can give measurements at least as accurate as ours.) I can't resist mentioning that this is another of the virtues of globular clusters: the narrowness of your main sequence is a measure of how well you have measured.

As an example, in the paper by Cool, Piotto, \& King, elsewhere in this volume, their Fig. 1 shows our color-magnitude diagram for NGC 6397, the globular cluster with the smallest apparent distance modulus. We get a remarkably narrow main sequence. Interestingly, the main sequence has striking bends, and it is a challenge for theories of stellar structure to match these. We have been in touch with some of the groups who calculate stellar models, and one of them has produced a quite good fit to our observations.

For a more extensive discussion of our results on NGC 6397, see Cool, Piotto, \& King (1996).

A really fascinating problem is the bottom of the main sequence. In the 
region near the faint limit of our observations is the mass where the lower limit of hydrogen burning should be. Although this point is well defined in mass, it is very ill defined in luminosity, because what happens here is that the luminosity plunges very steeply over a quite small range in mass. But luminosity is already very sensitive to mass even above the limit, so the luminosity function is already dropping rapidly and is thus hard to follow.

Getting the faint end of the luminosity function is even harder because of the presence of numerous field stars. At its low Galactic latitude $\left(b=-12^{\circ}\right)$, this cluster has a rich foreground, but with its moderate extinction $\left(A_{V}=\right.$ $0.56)$ it also has a rich background. Nature seems truly malicious here, as our best chance of locating the hydrogen-burning limit gets swamped in field stars. There is a way of solving the problem, however. The proper motion of the cluster is known, and we have calculated that by early 1997, a time for which HST observing proposals are already under review, the displacement of cluster stars from their 1994 positions will be great enough to allow a star-by-star separation of cluster from field. (In the course of demonstrating the accuracy of astrometry on WFPC2 images, we actually detected the mean motion of cluster stars between August and October 1994-another demonstration of the unprecedented accuracy that HST offers.)

The hydrogen-burning limit of course has very little to do with dynamics, but the number of low-mass stars does matter, and I will return to that.

Cool et al. also clearly show a white-dwarf sequence in NGC 6397 . The number of white dwarfs also matters to the dynamics, although our observations do not say much about that, because we see only the brightest white dwarfs, which are a small fraction of the total. Further study of white dwarfs will be of great interest to stellar-evolution theory, but for dynamical modeling we should continue to predict the WD number from assumptions about the mass function of the part of the main sequence that has already evolved away.

Another important result of the photometry is the luminosity function; for the dynamics of a cluster we certainly need to know the number of stars of each mass. Luminosity functions are discussed more extensively elsewhere in this volume by Piotto, Cool, \& King; so here I will merely mention that the derivation of LFs is far from trivial and that there are some unreliable results in the literature, particularly for the faint stars.

And even when one has a good luminosity function, there are further problems. For dynamical models one needs masses rather than luminosities, and the conversion is far from trivial. There is no observed mass-luminosity relation for the metallicity of this cluster $([\mathrm{Fe} / \mathrm{H}]=-1.9)$, and the theoretical M-L relations for low-mass metal-poor stars are far from reliable. As already mentioned, photometric results such as ours will stimulate and 


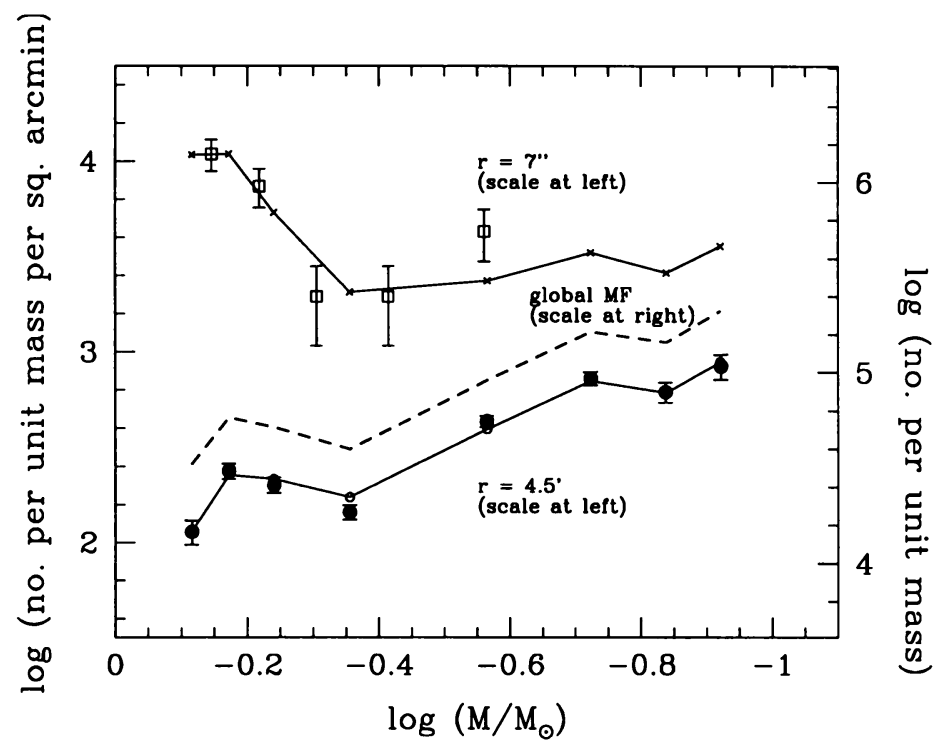

Figure 3. Mass functions in NGC 6397 at radii $7^{\prime \prime}$ and 4!6, as observed, in stars per arcmin $^{2}$. The numbers in the $7^{\prime \prime}$ field are higher because of the higher density at the cluster center, but the mass function is quite different. The broken lines are from a dynamical model fitted to the cluster; the numbers have been fitted to the observations at $4 ! 5$ but not at $7^{\prime \prime}$. Also shown is the global mass function of the model; the right-hand ordinate scale applies to it.

guide stellar-evolution theory, and the consequent improvements should result in better $\mathrm{M}-\mathrm{L}$ relations.

Even a perfect mass function would still leave one more problem: it is the local mass function of one region in the cluster, and what we need for dynamics is the global mass function of the cluster. For this a dynamical model is needed, and I will return to this question.

\section{Mass segregation}

Differences in the radial distributions of stars of different mass have been seen a number of times from the ground, significantly but weakly (Sandage 1954, Oort \& van Herk 1959, Richer \& Fahlman 1989, Drukier et al. 1993, and many other places). In HST images, however, we can see the faint stars all the way in to the cluster center, and the effects are tremendous. They have already been seen and commented on (Shara 1995; Paresce, De Marchi \& Jedrzejewski 1995). What we have done is to quantify the effect by fitting a cluster model (King, Sosin, \& Cool 1995).

Our first impression of the images of NGC 6397 was astonishment; there 
were almost no faint stars at the center. But when we fitted a dynamical model to the cluster, it became clear that this was just what was to be expected. Figure 3 shows the mass functions observed at the center and in an outlying field, and the fit of our theoretical model to them.

\section{Dynamic Modeling of NGC 6397}

For reasons that I have mentioned, we needed a dynamical model for NGC 6397. To create this I used a multi-mass embodiment of the algorithm that has given rise to the name "King models" (King 1966). In fitting the model, three conditions had to be satisfied. First, the model has to fit our observed mass function, when we project the model onto the plane of the sky at the radius at which our mass function was determined. Second, the central concentration of the model must match that of the cluster. Third, the projected luminosity profile of the model must agree in detail with that observed for the cluster.

The first two conditions were satisfied by trial and error. The third condition, however, introduced a separate aspect of cluster dynamics. The observed slope of the central part of the luminosity profile could be matched only by adding to the model a few hundred massive remnants. These stars have the effect of taking for themselves the -1 slope that belongs to a singular core of self-gravitating objects, so that in equipartition the red giants whose light we see have a flatter distribution. (This is a point that was made long ago by Lugger et al. [1987].) I arbitrarily assigned to these added objects masses of $1.4 \mathcal{M}_{\odot}$ and called them neutron stars, but a considerable trade-off is possible between their individual mass and their number, so that one cannot categorically identify them as neutron stars. No doubt central binaries figure in the picture too. What is certain, though, is that there are some hundreds of objects near the cluster center whose mass appreciably exceeds that of the red giants.

One thing that the reader may have noticed with surprise is that I have used a King model here to represent a cluster with a post-collapse core. This is a new thing; it has always been supposed that core collapse takes a cluster off the sequence that is represented by the King models. What I have done is simply to use a model with a high central concentration-so high, in fact, that its exact value doesn't matter; the center is essentially singular. The mathematical reason that this works is that the high-concentration limit of the King models is the original collapsed-core model of Hénon (1961); the physical reason is that a cluster as highly evolved as a post-core-collapse one has velocity distributions that are very close to Gaussian, and velocity dispersions that are rather close to Maxwellian. These are the essential characteristics of a King model. 


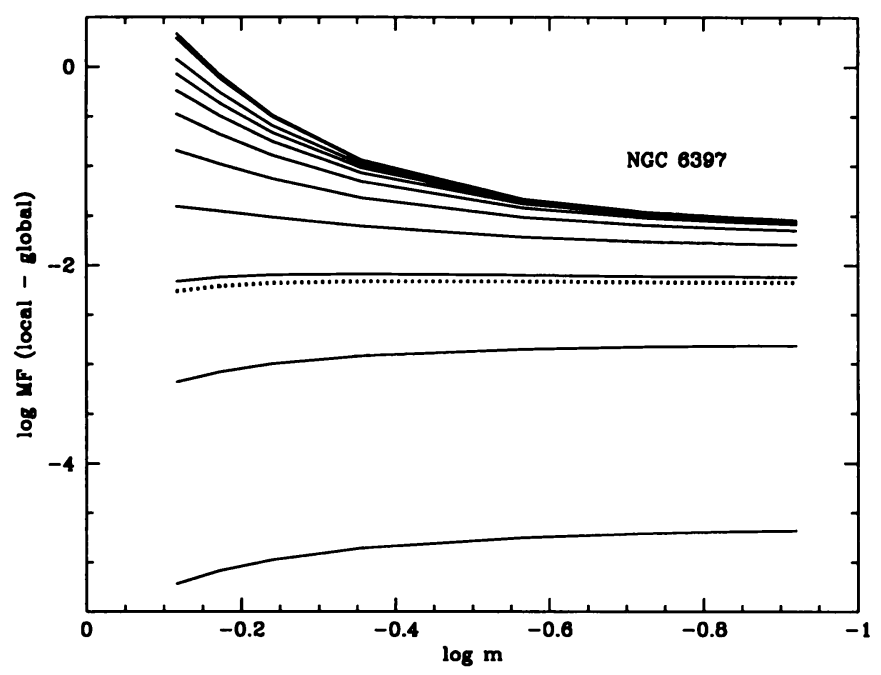

Figure 4. Differences between local mass functions at different radii (in projection) and the global mass function, in our model of NGC 6397. The dotted line represents the radius from which our observed MF came. The relative vertical positions of the curves reflect radial differences in density, but the zero point is arbitrary, since local and global MFs have different dimensions.

There are at least two reasons why King models of very high central concentration have not been explored. One is that beyond a central concentration of about 2.0 they have been shown to be unstable (Katz 1980, Wiyanto, Kato, \& Inagaki 1985); the other is that energetically a model cannot evolve along the sequence of King models beyond this point, because the magnitude of the potential energy no longer increases with increasing central concentration (King 1966). It is likely that the effect of binaries at the cluster center solves both of these problems.

Still another objection may be made to the model that I have used here. The Fokker-Planck integrations of Murphy, Cohn \& Hut (1990) showed that equipartition never quite catches up in a post-core-collapse cluster. The difference is quantitatively quite small, however; examination of one of their runs shows that a group with mass $0.14 \mathcal{M}_{\odot}$ has a velocity dispersion that would be appropriate for $0.18 \mathcal{M}_{\odot}$; I have not yet bothered to take this small difference into account, but easily could.

\section{Local vs. Global Mass Functions}

The availability of a cluster model makes it possible to convert from a mass function observed in one small part of the cluster to the global MF. Figure 4 illustrates the differences derived from our model of NGC 6397. The figure 

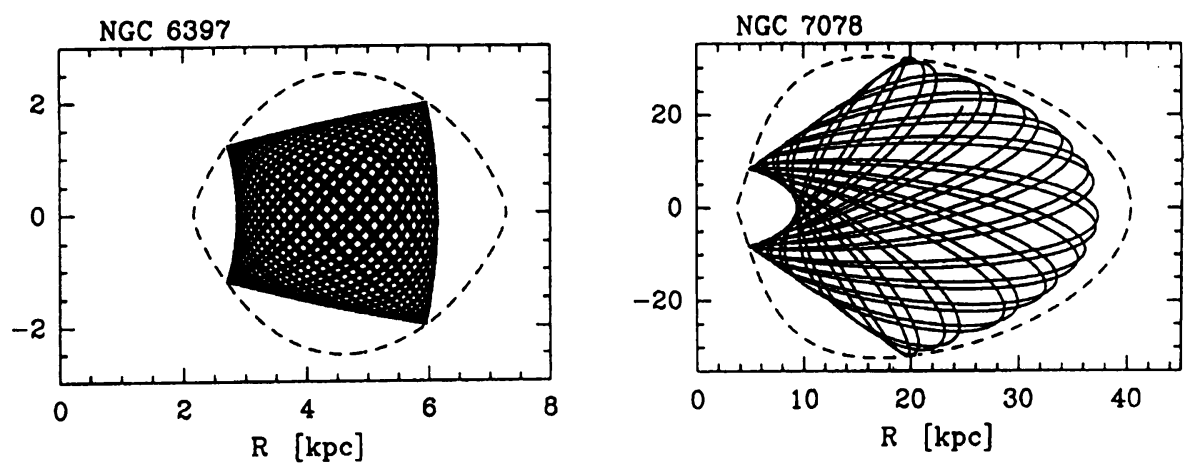

Figure 5. Comparison of the Galactic orbits calculated by Dauphole et al. 1995 for NGC 6397 and M15. NGC 6397's passages through the Galactic plane are much more frequent, and at lower inclination angles and smaller perigalacticon than those of M15, making it much more susceptible to star loss via tidal shocking.

again illustrates mass segregation, in that the higher-mass stars are greatly over-represented near the cluster center, relative to the less-massive stars, and they are under-represented near the edge. The figure also shows that the correction from local to global is relatively small over most of the outer part of the cluster.

\section{The Effect of Tidal Shocks}

Another interesting point about NGC 6397 is that the low-mass end of its luminosity function is deficient with respect to the LFs of other clusters of the same metallicity. The paper by Piotto, Cool, \& King elsewhere in this volume shows that the LFs of M15 and M30 are practically identical to each other, whereas NGC 6397 is strikingly poorer in faint stars. I emphasize: this cannot be a metallicity effect, because all three clusters have nearly the same metallicity.

As many people have suggested, the difference could be in the differing susceptibility of these clusters to tidal shocks. In Figure 5 are the Galactic orbits of NGC 6397 and of M15, in the meridional plane of $z$-coordinate and distance from the axis of rotation, as calculated by Dauphole et al. (1995). (Unfortunately the orbit of M30 is unknown.) Notice the differing scales of the two graphs. NGC 6397 is clearly hit much harder and much more often by passages through the Galactic plane. It is thus important in two ways that NGC 6397 stays so close to the Galactic center. First, it goes rapidly back and forth through the plane-a passage every 40 or $50 \mathrm{Myr}$. 
Second, the force exerted by the plane, which determines the strength of a shock, is much greater in there than farther out in the Galaxy.

But the effect of tidal shocks has never been quantified well enough, and Weinberg has shown (1994abc) that the traditional theories are not reliable. One project that we have under way is a numerical investigation in which we follow the orbits of a large number of representative stars in NGC 6397, as they move about in the cluster and the cluster moves in the Galactic gravitational potential.

\section{The Structure of Collapsed Cores}

Another problem that HST can illuminate is the structure of collapsed cores. Now for the first time we can see into these dense regions.

I will say rather little about this topic, however, because it is covered elsewhere in this volume by Sosin and King, who give the mass function and the radial density distribution near the center of M15.

One related point that I want to make is a somewhat sobering one, however. The FOC images that resolve the center of M15 are quite beautiful. But in some ways the situation is quite ugly. The core of M15 is so small that it does not have enough stars in it to trace its structure accurately. The trouble is that one cannot simply increase the statistical stability of the counts by including the numerous faint stars along with the brighter ones. The fainter stars have smaller masses, and therefore delineate a different density distribution. Hopefully this can be taken into account by more elaborate modeling, but the problem of the true nature of the core is clearly a more difficult one than can be solved by simply counting stars and drawing a profile.

There is one more matter about collapsed cores that I think deserves general attention. That is the question of stating the position of an object, such as an X-ray source, in terms of the number of core radii that it is from the center. This is perfectly proper for clusters that have a core, but it does not work for post-collapse clusters, most of which have nothing that can properly be called a core radius. The core radii that appear for these in the literature are meaningless force-fits of an inappropriate curve to some particular data set, and have little or nothing to do with the actual structure of the cluster center. (I myself confess to this sin [Trager, King, \& Djorgovski 1995], which I now regret very much.)

But it is nevertheless useful to have measure of how far out in the cluster the object is. Without a core radius, what can we use? There is a simple and obvious solution: state what fraction of the cluster's light lies within the cluster-centered circle that goes through the object in question. Then, to avoid using one datum for collapsed clusters and a different one for 
uncollapsed ones, I would suggest that the locations of objects such as Xray sources always be described by an "encircled fraction," and never by a number of core radii. The growth curves from which encircled fractions can be found are easily calculated from the cluster profiles given by Trager et al. (1995). (Their detailed data are indeed on the corresponding AAS CDROM, despite omission of the CD-ROM symbol in the table of contents.)

\section{Conclusion}

In considering the results that we and others are getting from HST, we should all remember that this is only a start. The results presented by several of us at this meeting all come from exposures taken in the first 15 months after the repair of the spherical aberration. As time goes on, HST will continue to contribute more and more to our understanding of the structure and dynamics of globular clusters.

\section{Acknowledgments}

It is a pleasure to recognize the collaboration of Adrienne Cool, Giampaolo Piotto, Craig Sosin, and Jay Anderson in the work that is described here. I also thank Andrea Lommen for orbital calculations and Charles Bartels for technical assistance. This work was supported by NASA grant NAG5-1607.

\section{References}

Cool, A. C., \& King, I. R. 1995, in Calibrating HST: Post Servicing Mission, ed. A. Koratkar \& C. Leitherer (Baltimore: STScI), p. 290

Cool, A. C., Piotto, G., \& King, I. R. 1996, ApJ, submitted

Dauphole, B., Geffert, M., Colin, J., Ducourant, C., Odenkirchen, M., \& Tucholke, H.-J. 1995, preprint

Drukier, G. A., Fahlman, G. G., Richer, H. B., Searle, L., \& Thompson, I. 1993, AJ, 106, 2335

Hénon, M. 1961, Ann.d'Ap., 24, 369

Katz, J. 1980, MN, 190, 497

King, I. R. 1966, AJ, 71, 64

King, I. R., Sosin, C., \& Cool, A. M. 1995, ApJL, 452, L33

Lugger, P. M., Cohn, H., Grindlay, J. E., Bailyn, C. D., \& Hertz, P. 1987, ApJ, 320, 482

Murphy, B. W., Cohn, H. N., \& Hut, P. 1990, MN, 245, 335

Oort, J. H., \& van Herk, G. 1959, BAN 14, 299 (No. 491)

Paresce, F., De Marchi, G., \& Jedrzejewski, R. 1995, ApJL, 442, 57

Richer, H. B., \& Fahlman, G. G. 1989, ApJ, 339, 178

Sandage, A. 1954, AJ, 59, 162

Shara, M. M., Drissen, L., Bergeron, L. E., \& Paresce, F. 1995, ApJ, 441, 617

Trager, S. C., King, I. R., \& Djorgovski, S. 1995, AJ, 109, 218

Weinberg, M. D. 1994a, AJ, 108, 1398

Weinberg, M. D. 1994b, AJ, 108, 1403

Weinberg, M. D. 1994c, AJ, 108, 1414

Wiyanto, P., Kato, S., and Inagaki, S. 1985, PASJ, 37, 715 